\title{
High-throughput Hyperspectral Microscopy
}

\author{
M.E. Gehm and D.J. Brady \\ Duke University Fitzpatrick Center for Photonics, Durham, NC, 27708
}

\begin{abstract}
A hyperspectral imager provides a 3-D data cube in which the spatial information (2-D) of the image is complemented by spectral information (1-D) about each spatial location. A static, high-throughput spectrometer design previously developed by our group can be used as the spectral engine in a high-throughput hyperspectral imager that avoids the Fourier undersampling issues present in previous dispersive designs. We present the theory for both pushbroom and tomographic operation and describe experimental results from our proof-of-concept implementation of a hyperspectral microscope.
\end{abstract}

Keywords: Hyperspectral imaging, multidimensional microscopy

\section{INTRODUCTION}

There is currently growing interest in a variety of multi-dimensional microscopy techniques throughout the bioscience communities. In this paper, we address hyperspectral microscopy. Traditional digital microscopy results in images with a scalar value attached to each pixel location. In hyperspectral microscopy, these scalar values are replaced with a vector containing the spectrum of the pixel. The resulting data cube is therefore three-dimensional (two spatial dimensions and one spectral dimension).

Historically, the applications requiring a spatial map of spectral variation have been largely limited to environmental remote sensing and military target discrimination. ${ }^{1,2}$ However, with the recent explosive growth of biomedical optics, hyperspectral imaging has come to be a major technique in that field, as well. ${ }^{3,4}$

Straightforward application of traditional hyperspectral techniques to biomedical systems, however, can be problematic. The simplest type of hyperspectral imager combines a tomographic (rotational scanning) or pushbroom (linear scanning) front-end with a traditional slit-based dispersive spectrometer. In biomedical optics, however, the sources tend to be weak and spatially-incoherent. Standard dispersive spectrometers have extremely poor photon collection efficiency for incoherent sources. When the source is also weak, the absolute number of collected photons can be very small. Further, this small number of photons must be apportioned amongst the large number of "cells" in the data cube. As a result, a given spatio-spectral element tends to contain very few photons and hence has a poor signal-to-noise ratio (SNR).

In response to this problem, the hyperspectral imaging community developed a number of different direct-view designs that maximize the light gathering efficiency of the systems. ${ }^{5-7}$ These systems do away with the spectrometer slit altogether and simply view the source through a rotating dispersive element. In this approach, the measurements taken at different rotation angles of the dispersive element are projective measurements through the data cube and can be tomographically reconstructed. While the photon efficiency of this type of approach is quite high, there is a drawback. The geometry of the system necessarily limits the range of angles over which projections are made. As a result of the Fourier-slice theorem, this yields an unsampled region of Fourier space. Consequently, the estimate of the data cube is inexact. In the tomographic community this Fourier undersampling is known as the missing cone problem, because the unsampled region is a conical volume in Fourier space.

Over the years there have been several other attempts to solve this problem. The two most notable are scanning-Michelson Fourier-transform spectrometers, and multiplexed pushbroom designs based on digital micromirror (DMM) technology. ${ }^{8,9}$ Both approaches are successful, however they also have some significant drawbacks. The Fourier-transform approach involves a scanning interferometer, and hence requires significant mechanical stability. The DMM approach has much in common with our pushbroom technique described below, but requires an expensive DMM array rather than a simple translation stage.

Three-Dimensional and Multidimensional Microscopy: Image Acquisition and Processing XIII, edited by Jose-Angel Conchello, Carol J. Cogswell, Tony Wilson, Proc. of SPIE Vol. 6090, 609007, (2006) 1605-7422/06/\$15 - doi: 10.1117/12.644828 
Our group has developed a class of static, coded-aperture dispersive spectrometers designed for working with the weak, incoherent sources common to biomedical applications. ${ }^{10,11}$ In addition to working as general spectrometers, these instruments can serve as the spectral engine in a hyperspectral imager. The instrument achieves a powerful compromise - there is no Fourier undersampling, yet the photon collection efficiency is only a factor of 2 less than the direct-view system (still orders of magnitude improvement over slit-based spectral engines).

This paper discusses the theory behind the application of these spectral engines to hyperspectral imaging and provides first proof-of-concept results from a hyperspectral microscope we have constructed using these ideas.

\section{SYSTEM THEORY}

This section addresses the underlying theory of our hyperspectral imager. The discussion will focus on how our coded-aperture spectral engine can easily be transformed into a mechanically-robust, high-throughput hyperspectral imager that does not suffer from the missing-cone problem. For full details on the theory and operation of the coded-aperture spectrometer, the reader is referred to Ref. 10.

\subsection{MEASUREMENT DIVERSITY}

To reconstruct a three-dimensional volume from measurements on a two-dimensional detector plane requires either a time series of measurements or the reduction of the reconstructed volume such that the full series of measurements can be tiled on the available detector array. We refer to these two methods as temporallysequenced and spatially-sequenced respectively. In the remainder of this paper we discuss only temporallysequenced systems, although the arguments also hold in general for spatially-sequenced systems.

Of course, a series of non-varying measurements is no more informative than a single measurement (ignoring issues of SNR). To provide diversity between the measurements, the system is scanned (in the spatially-sequenced system, the tiled measurements are diverse by design). We consider both linearly-scanned (pushbroom) and rotationally-scanned (tomographic) systems.

\subsection{SPECTRAL ENGINE}

We can model the intensity on the detector plane of our coded-aperture spectrometer as

$$
I\left(x^{\prime}, y^{\prime}\right)=\iiint d \lambda d x d y \delta\left(y-y^{\prime}\right) \delta\left(x-\left(x^{\prime}+\alpha\left(\lambda-\lambda_{c}\right)\right)\right) T(x, y) S(x, y ; \lambda) .
$$

Here $T(x, y)$ is the transmission pattern of the aperture code, $S(x, y ; \lambda)$ is the spectral density of the source at the input aperture (the data cube we hope to estimate), and the Dirac delta functions describe the propagation through a unity-magnification dispersive spectrometer with linear dispersion $\alpha$ and a center wavelength of $\lambda_{c}$ for an aperture located at $x=0$. Performing the $\lambda$ and $y$ integrals results in

$$
I\left(x^{\prime}, y^{\prime}\right)=\int d x T\left(x, y^{\prime}\right) S\left(x, y^{\prime} ; \frac{x-x^{\prime}}{\alpha}+\lambda_{c}\right) .
$$

We then form an estimate by multiplying with an analysis function $\tilde{T}\left(x^{\prime \prime}, y^{\prime}\right)$ and integrating over the full range of $y^{\prime}$

$$
\begin{aligned}
E\left(x^{\prime \prime}, x^{\prime}\right) & =\int_{y_{\min }^{\prime}}^{y_{\max }^{\prime}} d y^{\prime} \tilde{T}\left(x^{\prime \prime}, y^{\prime}\right) I\left(x^{\prime}, y^{\prime}\right) \\
& =\int_{y_{\min }^{\prime}}^{y_{\max }^{\prime}} d y^{\prime} \tilde{T}\left(x^{\prime \prime}, y^{\prime}\right) \int d x T\left(x, y^{\prime}\right) S\left(x, y^{\prime} ; \frac{x-x^{\prime}}{\alpha}+\lambda_{c}\right) .
\end{aligned}
$$

At this point we can go no further in our analysis unless the source $S$ is approximately uniform in $y$,

$$
S(x, y ; \lambda) \approx I(y) S(x ; \lambda) .
$$


When this condition holds, and $T(x, y)$ and $\tilde{T}(x, y)$ are properly designed (see Ref. 10), $E\left(x^{\prime \prime}, x^{\prime}\right)$ becomes an estimate of the one-dimensional spectral density of the source

$$
E\left(x^{\prime \prime}, x^{\prime}\right) \approx \beta S\left(x^{\prime \prime} ; \frac{x^{\prime \prime}-x^{\prime}}{\alpha}+\lambda_{c}\right) .
$$

The sections below detail how to use a series of measurements of this form to reconstruct the full data cube.

\subsection{PUSHBROOM OPERATION}

In the pushbroom system design, we use a linear scan to provide measurement diversity. Between measurements, the source is shifted in $y$ relative to the input aperture of the spectrometer. We can generalize Eq. 1 to include the shift,

$$
I\left(x^{\prime}, y^{\prime}, \Delta\right)=\iiint d \lambda d x d y \delta\left(y-y^{\prime}\right) \delta\left(x-\left(x^{\prime}+\alpha\left(\lambda-\lambda_{c}\right)\right)\right) T(x, y) S(x, y-\Delta ; \lambda) .
$$

When considered over a range of $\Delta$ values, this becomes a three-dimensional data set. As before, we perform the $\lambda$ and $y$ integrals,

$$
I\left(x^{\prime}, y^{\prime}, \Delta\right)=\int d x T\left(x, y^{\prime}\right) S\left(x, y^{\prime}-\Delta ; \frac{x-x^{\prime}}{\alpha}+\lambda_{c}\right) .
$$

Unfortunately, for an arbitrary source, the approximation of Eq. 4 does not hold.

To proceed, we consider the two-dimensional intensity profile in a plane where $y^{\prime}-\Delta$ takes on the constant value $p$.

$$
\begin{aligned}
I_{p}\left(x^{\prime}, y^{\prime}\right) & =\int d \Delta \delta\left(\Delta-\left(y^{\prime}-p\right)\right) I\left(x^{\prime}, y^{\prime}, \Delta\right) \\
& =\int d x T\left(x, y^{\prime}\right) S\left(x, p ; \frac{x-x^{\prime}}{\alpha}+\lambda_{c}\right) .
\end{aligned}
$$

However, since $p$ is a constant,

$$
S\left(x, p ; \frac{x-x^{\prime}}{\alpha}+\lambda_{c}\right)=I(p) S\left(x, \frac{x-x^{\prime}}{\alpha}+\lambda_{c}\right) .
$$

In these planes, the approximation of Eq. 4 not only holds, it is exact. We can therefore use the result of Eq. 5 to write

$$
E_{p}\left(x^{\prime \prime}, x^{\prime}\right)=\beta S\left(x^{\prime \prime} ; \frac{x^{\prime \prime}-x^{\prime}}{\alpha}+\lambda_{c}\right) .
$$

If we then calculate an estimate of this form for all possible values of $p$, we can layer the results to form the three-dimensional data set

$$
E\left(x^{\prime \prime}, p, x^{\prime}\right)=\beta S\left(x^{\prime \prime}, p ; \frac{x^{\prime \prime}-x^{\prime}}{\alpha}+\lambda_{c}\right),
$$

which is directly proportional to the data cube we wished to measure.

Heuristically, it is easy to understand how this procedure works. A source with structure in $y$ cannot meet the uniformity requirement needed for processing. However, working in a plane where $y^{\prime}-\Delta$ is constant is equivalent to piecing together an intensity profile by selecting rows from each measurement that correspond to only a single given $y$-coordinate in the source (the coordinate $y=p$ in the image taken with no shift). Thus, by construction, these intensity profiles have no variation in $y$. These planes can then be processed according to the normal procedure and then layered to build up information about the $y$ structure of the source. 


\subsection{TOMOGRAPHIC OPERATION}

In the tomographic system design, measurement diversity is provided by a rotational scan. However, there is a difficulty. The approximation of Eq. 4, does not hold in general. Further, unlike the pushbroom case, there are no planes in the collected data set where it does hold. Instead, we use optical elements located prior to the input aperture of the spectral engine to manipulate the optical fields into a form compatible with Eq. 4 . Later sections discuss the optical elements used to achieve these transformations.

We define the spectral source prior to any manipulation as $S_{\text {orig }}(x, y ; \lambda)$. The first step of optical processing is to rotate the source in the $x-y$ plane

$$
S_{\text {rot }, \theta}(x, y ; \lambda)=S_{\text {orig }}(x \cos \theta-y \sin \theta, y \cos \theta+x \sin \theta ; \lambda) .
$$

We then use an astigmatic optical element to eliminate the structure in $y$.

$$
S_{\text {final }, \theta}(x ; \lambda) \approx \int d y S_{\text {rot }, \theta}(x, y ; \lambda) \approx \int d y S_{\text {orig }}(x \cos \theta-y \sin \theta, y \cos \theta+x \sin \theta ; \lambda) .
$$

The source profile $S_{\text {final, } \theta}$ does satisfy the requirements of Eq. 4, and we can proceed as before. However, before proceeding we take a moment to note that the operations of Eq. 13 are equivalent to the definition of the Radon transform $\mathcal{R}_{x, \theta}$. That is,

$$
S_{\text {final }, \theta}(x ; \lambda) \approx \mathcal{R}_{x, \theta}\left[S_{\text {orig }}(x, y ; \lambda)\right]
$$

If we allow $S_{\text {final, } \theta}$ to act as the source for the spectral engine, we can proceed as in Eq. 5 and process the measured intensity profile into an estimate of the input spectrum

$$
E_{\theta}\left(x^{\prime \prime}, x^{\prime}\right) \approx \beta S_{\text {final }, \theta}\left(x^{\prime \prime} ; \frac{x^{\prime \prime}-x^{\prime}}{\alpha}+\lambda_{c}\right) .
$$

If we perform this procedure over a range of angles $\theta$, we can construct a three-dimensional data set which is approximately the Radon transform of the original data cube:

$$
E\left(x^{\prime \prime}, \theta, x^{\prime}\right) \approx \beta S_{\text {final }, \theta}\left(x^{\prime \prime} ; \frac{x^{\prime \prime}-x^{\prime}}{\alpha}+\lambda_{c}\right) \approx \beta \mathcal{R}_{x^{\prime \prime}, \theta}\left[S_{\text {orig }}\left(x^{\prime \prime}, y ; \frac{x^{\prime \prime}-x^{\prime}}{\alpha}+\lambda_{c}\right)\right] .
$$

An estimate of the original data cube can then be constructed by taking the inverse Radon transform of the data set

$$
S_{\text {orig }}\left(x^{\prime \prime}, y ; \frac{x^{\prime \prime}-x^{\prime}}{\alpha}+\lambda_{c}\right) \approx \mathcal{R}_{x^{\prime \prime}, \theta}^{-1}\left[\frac{1}{\beta} E\left(x^{\prime \prime}, \theta, x^{\prime}\right)\right] \text {. }
$$

Heuristically, the system can be viewed as measuring projections through the data cube in directions perpendicular to the $\lambda$-axis. These projections can be reconstructed into the underlying data cube using the standard processing methods of parallel-beam tomography. Because the projection angles are measured around the $\lambda$-axis, there are no limitations to the range of $\theta$, and the system can fully sample the Fourier space. This is in contrast to direct-view tomographic methods where the projection style is analogous to cone-beam tomography, and the size of the input aperture limits the range of possible angles - resulting in the missing-cone problem of Fourier undersampling.

\section{SYSTEM DESIGN}

To test these hyperspectral imaging ideas, we have constructed a proof-of-concept hyperspectral microscope system. This section describes the optical and mechanical features of the prototype system. A schematic of the system is shown in Fig. 1 


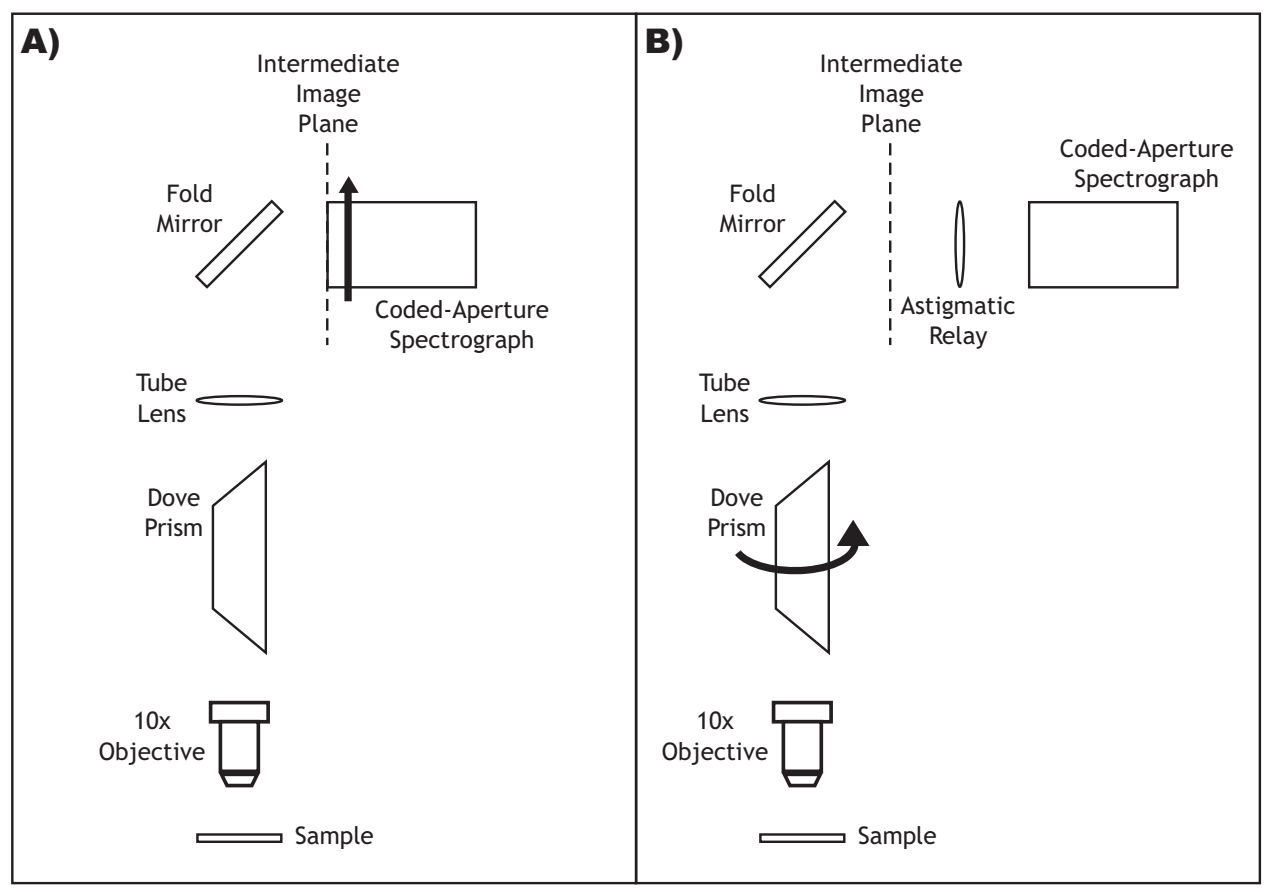

Figure 1. A schematic of the proof-of-concept hyperspectral microscope. In A), the system is configured for pushbroom operation. In B), the system is configured for tomographic operation.

\subsection{MICROSCOPE FRONT END}

The microscope front end is the portion of the optical path running from the sample to the intermediate image plane. The composition of the front end is identical in both pushbroom and tomographic configurations.

The front end begins with a Nikon $10 \times$ infinity-corrected microscope objective. The Nikon objective was chosen because it was fully achromatized, and did not require a matched tube lens for color-free performance.

In the collimated space immediately following the objective, we place a dove prism. A dove prism is an optical element that rotates an optical field by an angle $2 \theta$ when the prism is rotated by an angle $\theta$ about its axis. In the tomographic configuration, we rotate the dove prism to achieve the various rotated optical fields required by the technique. In the pushbroom configuration, the prism remains fixed.

Next in the optical path is a $250 \mathrm{~mm}$ focal-length acromat as the tube lens. This lens forms an image of the sample in the intermediate image plane. The image plane occurs after a fold mirror which is used to provide a more compact system. The intermediate image plane marks the termination of the microscope front end.

\subsection{ASTIGMATIC RELAY}

In the tomographic configuration, we require the input distribution to be approximately uniform in the $y$-direction (see Sec. 2.4). This is accomplished with the astigmatic relay section. This is a set of lenses designed such that, in the $x$-direction, the intermediate image plane is imaged onto the input aperture of the spectral engine, while the $y$-direction is Fourier transformed. For most natural sources, this has the effect of eliminating the structure in $y$ precisely as desired.

When the system is operated in the pushbroom configuration, this relay section is removed.

\subsection{CODED APERTURE SPECTROMETER}

The spectral engine is a compact version of the static multimodal multiplex spectrometer design described in Refs. 10 and 11. The spectral engine was built from on-hand optical components. This resulted in a spectral range of approximately $550-665 \mathrm{~nm}$. The aperture code used is an order- 64 S-matrix ${ }^{10}$ with 6 pixels per mask element. 


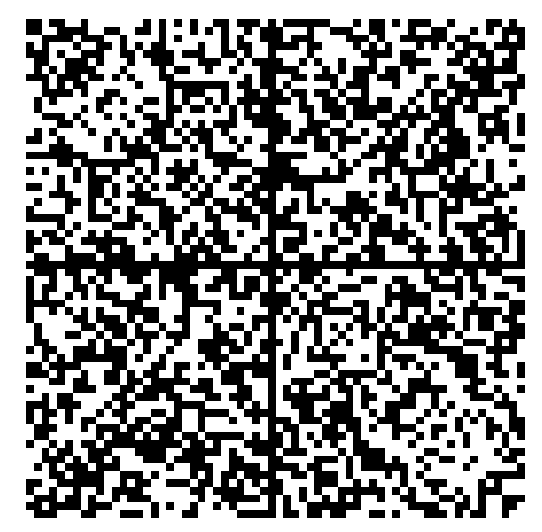

Figure 2. The aperture coding pattern used in the spectral engine. The structure is an order-64 S-matrix with the rows and columns randomly permuted to remove spurious correlations.

The aperture pattern is shown in Fig. 2. The resulting spectral resolution of the instrument is approximately 1 nm.

\subsection{MECHANICAL STRUCTURE AND SCANNING SYSTEMS}

The majority of the mechanical structure of the instrument was designed in a 3D computer-aided-design (CAD) program and "printed" on a rapid-prototyping machine. The material used is a UV-cure polymer with acrylic-like mechanical and thermal properties.

The individual subsystems (front end, astigmatic relay, and spectral engine) are mounted on several large aluminum support posts. The post for the spectral engine is placed on a computer-controlled translation stage. During pushbroom operation, this stage is activated to scan the input aperture of the spectral engine across the intermediate image plane formed by the microscope front end. The dove prism is mounted in a manuallycontrolled rotation stage. During tomographic operation, this stage is adjusted by hand to achieve the desired rotation of the source field in the intermediate image plane.

\section{EXPERIMENTAL TEST AND RESULTS}

To test the performance of the proof-of-concept system we used it to estimate the data cube for a simple sourcea chrome-on-glass transmission target illuminated from underneath with a diffused helium-neon (HENE) laser. The transmission pattern used is shown in Fig. 3. The smallest feature size on the mask is approximately 3 microns square. For a source of this type, the data cube is a empty except for a single plane corresponding to the laser wavelength and having the spatial structure of the transmission mask. The remainder of this section presents our initial results for the two system configurations.

\subsection{PUSHBROOM TEST}

For the pushbroom experiment, the system was used to make 128 distinct measurements. Between measurements, the computer controlled translation stage moved the spectral engine 54 microns (1 row on the aperture code) in $y$ with respect to the intermediate image plane. The data were processed according to the description in Sec. 2.3. Results from the experiment are shown in Fig. 4.

The results demonstrate an impressive SNR. The image on the left is produced by summing through the entire data cube and corresponds to an intensity image of the source. Fine features are clearly visible (including the fact that the transmission mask was not uniformly illuminated by the laser). The image on the right is produced by summing through only a few spectral bands - those corresponding to a narrow wavelength range centered on the HeNE laser. The SNR in this case is quite impressive, and in comparison with the image from the full 


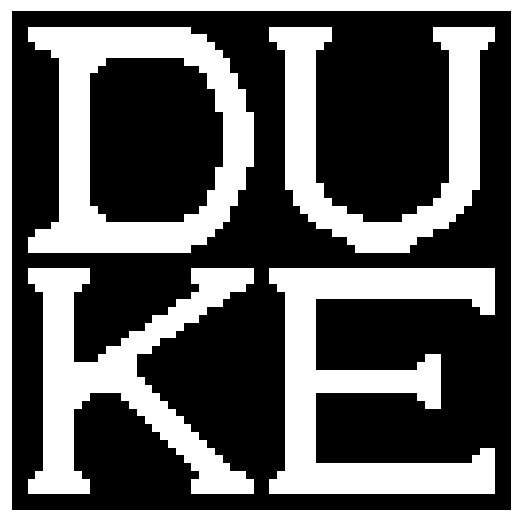

Figure 3. A plot of the transmission mask used to generate the optical source. The pattern is a $64 \times 64$ discrete grid of regions that are either $100 \%$ transparent or $100 \%$ opaque.
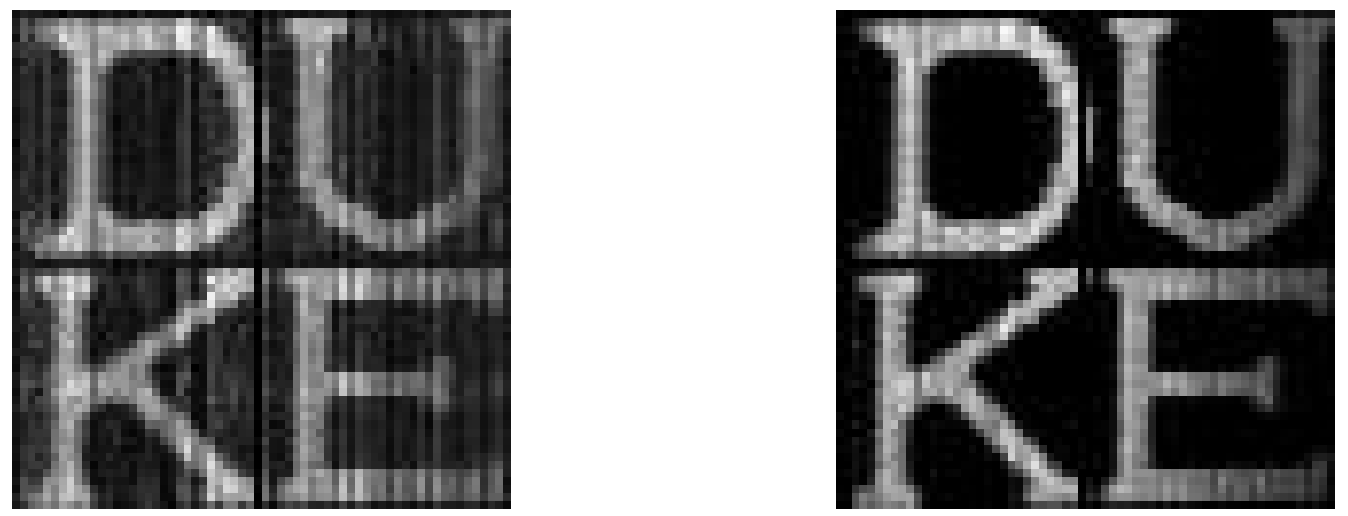

Figure 4. Results from the pushbroom experiment. The image on the left is the sum through the entire reconstructed data cube - corresponding to an intensity image of the source. The image on the right is a sum through only a few spectral bands centered around the HENE laser wavelength. The SNR is quite good in both cases. The dark vertical and horizontal bands crossing in the center of the images are alignment marks placed in the aperture code of the spectral engine (and can be seen in Fig. 2). They would not be present in a final design. 


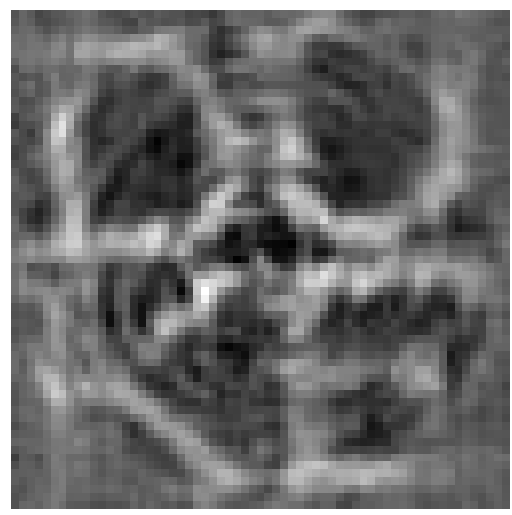

Figure 5. Results from the tomographic experiment. The image is the sum through a few spectral bans centered around the HENE laser wavelength. Possible reasons for the poor SNR are discussed in the text.

data cube reveals that there must be a small amount of noise in the regions of the data cube which should be empty. An animation of spectral slices through the reconstructed data cube does indeed show a small amount of reconstruction noise throughout the cube. The reconstructions also contain a dark vertical and horizontal band crossing near the center of the images. These are alignment marks that were placed in the aperture code of the spectral engine and are not reconstruction artifacts. The marks are clearly visible in Fig. 2. They alignment marks would be removed in a final system.

There is one further issue that is not easily shown in a manuscript. The image of "DUKE" in the reconstructed data cube is not perfectly orthogonal to the $\lambda$-axis. This is equivalent to a disagreement about the laser wavelength from the left to right side of the image. This effect likely results from slight errors in the calibration process and should be correctable with improved algorithms.

\subsection{TOMOGRAPHIC TEST}

For the tomographic experiment, the system was used to make 179 distinct measurements. Between measurements, the manual rotation stage containing the dove prism was rotated by $1^{\circ}$. Because the dove prism rotates the optical field by twice the applied rotation, the optical field was rotated in $2^{\circ}$ increments from $0-358^{\circ}$. The data were processed according to the description in Sec. 2.4 using the standard inverse Radon technique of filtered back-projection. Results from the experiment are shown in Fig. 5.

The results of the tomographic experiment are not good, although it is possible to recognize the source in the reconstructed image. We believe that there are two primary experimental causes for the poor tomographic results. First, the manual rotation stage is inaccurate and difficult to control at this resolution. The printed scale on the stage is in $2^{\circ}$ increments. Providing $1^{\circ}$ steps manually without a feedback system likely introduces errors that are large compared to the desired step-size. Second, the process of touching the instrument to adjust the stage likely results in non-repeatable shifts in the mechanical structure of the microscope front end. This mechanical structure is made of rapid-prototyping plastic and is much more flexible than a similar metal structure would be. Transverse motions of the objective as small as 3 microns are sufficient to provide shifts of one spatial resolution element. The validity of these two arguments is bolstered by the fact that the pushbroom results improved dramatically with the conversion from manual to computer-controlled actuation.

\section{FUTURE PLANS AND SUMMARY}

There are two main thrusts of work remaining on this project. First, we plan to transition to a computercontrolled rotation stage in the near future. A stage has been ordered and we are awaiting delivery. 
A more substantial modification involves the ability to work with more complicated sources. The intensity profile captured on the detector plane of the spectral engine is a complicated combination of the source spatial structure, source spectrum, and the aperture coding pattern. For the simple laser illumination described above, a sharp image of the source pattern modulated by the input aperture pattern is observed. This is suitable for focusing the instrument on the sample plane. However, for more complicated sources, the observed intensity pattern is a complicated convolution without any apparent spatial structure that could reveal proper focus. As a result, attempts to work with more complicated source have not been successful. We plan on modifying the instrument by replacing the fold mirror with a high-reflectivity beam-splitter. A second detector array will then be placed above the fold mirror and used to determine when the sample is properly focused.

In summary, we have developed a basic theory that shows how the static aperture-coded spectrometer design developed by our group ${ }^{10,11}$ can be used for extremely high-thoughput hyperspectral imaging without Fourier undersampling issues. Further, we have constructed a proof-of-concept testbed for experimental validation of these claims. For a relatively simple source, the pushbroom configuration performs admirably, recovering the full data cube with high SNR. The tomographic reconstruction is recognizable as the source, however the quality is not sufficient to yet justify the claim about a lack of undersampling. The poor performance is blamed largely on mechanical issues relating to the manual rotation of the dove prism. Improvements are planned to rectify the shortcoming in the tomographic approach and to allow the system to work with more complicated sources.

\section{ACKNOWLEDGMENTS}

The authors would like to acknowledge B.D. Guenther and S.T. McCain for stimulating discussions on this topic. This work was supported by the AFOSR through grant \#F49620-02-1-0335.

\section{REFERENCES}

1. W. Smith, D. Zhou, F. Harrison, H. Revercomb, A. Larar, A. Huang, and B. Huang, "Hyperspectral remote sensing of atmospheric profiles from satellites and aircraft," Proc. SPIE 4151, pp. 94-102, 2001.

2. C. Stellman, F. Olchowski, and J. Michalowicz, "WAR HORSE (wide-area reconnaissance: hyperspectral overhead real-time surveillance experiment)," Proc. SPIE 4379, pp. 339-346, 2001.

3. T. Pham, F. Bevilacqua, T. Spott, J. Dam, B. Tromberg, and S. Andersson-Engles, "Quantifying the absorption and reduced scattering coefficients of tissuelike turbid media over a broad spectral range with noncontact fourier-transform hyperspectral imaging," Appl. Opt. 39, pp. 6487-6497, 2000.

4. R. Schultz, T. Nielsen, J. Zavaleta, R. Ruch, R. Wyatt, and H. Garner, "Hyperspectral imaging: A novel approach for microscopic analysis," Cytometry 43, pp. 239-247, 2001.

5. M. Descour and E. Dereniak, "Computed-tomography imaging spectrometer: experimental calibration and reconstruction results," Appl. Opt. 34, pp. 4817-4826, 1995.

6. P. Bernhardt, "Direct reconstruction methods for hyperspectral imaging with rotational spectrotomography," J. Opt. Soc. Am. A 12, pp. 1884-1901, 1995.

7. J. Mooney, V. Vickers, M. An, and A. Brodzik, "High-throughput hyperspectral infrared camera," J. Opt. Soc. Am. A 14, pp. 2951-2961, 1997.

8. C. Snively, G. Katzenberger, and J. Lauterbach, "Fourier-transform infrared imaging using a rapid-scan spectrometer," Opt. Lett. 24, pp. 1841-1843, 1999.

9. A. Wuttig and R. Riesenberg, "Sensitive Hadamard transform imaging spectrometer with a simple MEMS," Proc. SPIE 4881, pp. 167-178, 2003.

10. M. Gehm, S. McCain, N. Pitsianis, D. Brady, P. Potuluri, and M. Sullivan, "Static 2D aperture coding for multimodal multiplex spectroscopy," Appl. Opt. , May 2006. To appear.

11. S. McCain, M. Gehm, Y. Wang, N. Pitsianis, and D. Brady, "Coded aperture Raman spectroscopy for quantitative measurements of ethanol in a tissue phantom," Submitted to Applied Spectroscopy. 